\title{
The Effect of Lesions of the Basolateral Amygdala on Instrumental Conditioning
}

\author{
Bernard W. Balleine, ${ }^{1}$ A. Simon Killcross, ${ }^{2}$ and Anthony Dickinson ${ }^{3}$ \\ ${ }^{1}$ Department of Psychology and the Brain Research Institute, University of California Los Angeles, Los Angeles, California 90095-1563, ${ }^{2}$ School of \\ Psychology, University of Wales, Cardiff, United Kingdom CF10 3XQ, and ${ }^{3}$ Department of Experimental Psychology, University of Cambridge, Cambridge \\ United Kingdom CB2 3EB
}

In three experiments, we assessed the effect of lesions of the amygdala basolateral complex (BLA) on instrumental conditioning in rats. In experiment 1, the lesion had no effect on the acquisition of either lever pressing or chain pulling in food-deprived rats whether these actions earned food pellets or a maltodextrin solution. The lesion did attenuate, however, the impact of outcome devaluation, induced by sensory-specific satiety, on instrumental performance both when assessed in extinction and when reward was delivered contingent on instrumental performance. In experiment 2, evidence was found to suggest that the lesioned rats differed from shams in their ability to encode the specific action-outcome contingencies to which they were exposed during training: lesioned rats failed to adjust their performance appropriately when the action-outcome contingency was degraded. These effects were not caused by an inability of BLA lesioned rats to discriminate the two instrumental actions; these rats were similar to shams in their acquisition of a heterogeneous instrumental chain involving lever pressing and chain pulling (experiment 3 ). In experiment 4 , however, lesions of the BLA were found to produce a deficit in the ability of rats to use the specific properties of the instrumental outcomes used in the previous experiments to discriminate rewarded from unrewarded actions in a free operant discrimination situation. Together these results suggest that in instrumental conditioning, the BLA mediates outcome encoding, specifically relating the sensory features of nutritive commodities to the emotional consequences induced by their consumption.

Key words: instrumental conditioning; basolateral nucleus; amygdala; reinforcer devaluation; sensory specific-satiety; incentive learning; contingency; reward

\section{Introduction}

When hungry rats are trained to lever press to gain access to food, evidence suggests that they encode an association between the action (lever pressing) and quite specific sensory features of that nutritive outcome (Rescorla, 1990; Balleine and Dickinson, 1998a,b, 2000). In addition, it is now well established that the rats' evaluation of the incentive value of the food, i.e., its affective and motivationally relevant properties, critically determines the performance of actions instrumental to its delivery (for review, see Dickinson and Balleine, 1994; Balleine, 2001). Evidence for this claim has come mainly from studies assessing the impact of shifts in primary motivation on instrumental performance. Posttraining shifts, such as from hunger to satiety, often have very little direct impact on instrumental performance unless the effect of this shift on the incentive value of nutritive events is made explicit through consummatory experience, i.e., through incentive learning (Dickinson and Balleine, 1994). With regard to instrumental responding for food, therefore, satiety acts to reduce performance, not because it reduces drive (Hull, 1943), but because it reduces the value of nutritive outcomes (cf. Balleine, 1992, 2001; Balleine and Dickinson, 1994).

Received July 8, 2002; revised 0ct. 16, 2002; accepted 0ct. 22, 2002.

This research was supported by grants from the National Institute of Mental Health (MH56446) and the European Commission Biomed 2 Program.

Correspondence should be addressed to Bernard W. Balleine, Department of Psychology, University of California, Los Angeles, Box 951563, Los Angeles, CA 90095-1563. E-mail: balleine@psych.ucla.edu.

Copyright $\odot 2002$ Society for Neuroscience $\quad 0270-6474 / 02 / 220666-10 \$ 15.00 / 0$
This effect of satiety on instrumental performance can be highly specific. Thus, when hungry rats are exposed to a specific satiety procedure in which they are given the opportunity to consume a particular food for an extended period, they subsequently reduce their performance of actions that gain access to that specific food essentially without any effect on the performance of actions that gain access to a different food (Balleine and Dickinson, 1998a,b, 2000; Corbit and Balleine, 2000; Corbit et al., 2001). Furthermore, devaluation by sensory-specific satiety can be found when two nutritive outcomes differ only in a single taste feature (Balleine and Dickinson, 1998a; Corbit and Balleine, 2000), indicating that devaluation affects the taste component of the food and suggesting that the neural processes involved in the detection and representation of taste may be critically involved in encoding changes in the incentive value of nutritive events.

Recently, this claim was assessed by examining the effect of lesions of the gustatory region of the insular cortex (GC) on instrumental conditioning in rats (Balleine and Dickinson, 2000). This lesion was found to attenuate the impact of sensoryspecific satiety on instrumental performance but only when assessed in an extinction test. The lesion had no effect on specific satiety-induced devaluation when the rewards were delivered nor did it have any detectable effect on the rats' ability to encode the specific contingency between its actions and their consequences.

These data were interpreted by Balleine and Dickinson (2000) as indicating that the GC operates as one component of an incentive system, acting to encode the taste features of the instrumental outcome as an aspect of the representation of that outcome in 
memory. From this perspective, the GC is not involved in detecting changes in incentive value; that would appear to require the integration of taste memory, involving the GC, with an affective signal, apparently mediated by a different component of the incentive system (cf. Balleine, 2001). In this regard, it is worth noting that the GC maintains strong reciprocal connections with the amygdala (Sripanidkulchai et al., 1984; Yamamoto et al., 1984), a connection that has been implicated in taste-affect integration in taste aversion learning (Gallo et al., 1992). Assessing the role of the amygdala in instrumental conditioning would appear to provide, therefore, an important step in further investigating the neural bases of incentive processes.

In this series of experiments, procedures similar to those described by Balleine and Dickinson (2000) were used to assess the impact of lesions of the BLA on instrumental learning and performance. After recovery from surgery, we first compared the acquisition of lever pressing and chain pulling in lesioned and sham-lesioned rats in a situation in which one action earned access to food pellets and the other to a maltodextrin solution. Acquisition was followed by further instrumental training sessions before a series of three tests designed to assess the ability of lesioned rats to encode specific action-outcome associations as well as the action-outcome contingency. The ability of BLA lesioned rats to discriminate the instrumental actions and outcomes used in these initial tests was assessed in two further experiments. First, we assessed the ability of rats to acquire a chain of lever press and chain pull actions, requiring them to perform the two actions in a prescribed sequence to gain access to reward (cf. Balleine et al., 1995). Subsequently, rats were trained on a task requiring them to use the specific sensory properties of the instrumental outcomes to discriminate rewarded from unrewarded actions in a free operant discrimination situation.

\section{Materials and Methods Experiment 1}

\section{Subjects and apparatus}

Subjects were 16 male Hooded Lister rats (OLAC, Bicester, UK). They were housed in squads of four in a temperature- and humiditycontrolled room with lights on from 8 A.M. to 8 P.M. After delivery, rats were maintained on ad libitum food and water and operated on when they weighed at least $300 \mathrm{gm}$. After surgery, the animals were returned to their home cages for a $10 \mathrm{~d}$ period of recuperation. At this point, and for all stages of behavioral testing, the animals were shifted to a $22.5 \mathrm{hr}$ food deprivation schedule under which they received access to food for $1.5 \mathrm{hr}$ each day in their home cage at least $2 \mathrm{hr}$ after behavioral testing, with water available continuously.

Instrumental training and testing were conducted in four Campden Instruments (Manchester, UK) operant chambers. Each chamber was equipped with a recessed magazine, a retractable lever, and a chain. The magazine was positioned in the center of the front wall and could be entered via a flap door, which was attached to a microswitch. The lever and chain (which was lowered through the ceiling from a microswitch) were positioned symmetrically to the right and left side, respectively, of the magazine flap. The chambers were also fitted with a pellet dispenser and a peristaltic pump, both of which were programmed to deliver the instrumental outcomes into the recessed magazine. The outcomes used were a $45 \mathrm{mg}$ Noyes pellet (formula A) and $0.05 \mathrm{ml}$ of a $20 \%$ solution of maltodextrin (Cerestar, Manchester, UK). Each chamber was illuminated by a $3 \mathrm{~W}$ house light mounted in the center of the front panel above the magazine. A BBC microcomputer equipped with the SPIDER extension for on-line control (Paul Fray Ltd., Cambridge, UK) controlled the equipment and recorded lever presses and chain pulls. For the presentation of the outcomes outside the operant chambers, eight feeding cages were used. These were molded plastic boxes, $30 \times 13 \times 11 \mathrm{~cm}$ in size, with wire mesh ceilings. Pellets were given in small glass dishes placed inside these cages, whereas maltodextrin was given through calibrated drinking tubes inserted through a hole in the wire mesh ceiling.

\section{Surgical procedures}

Animals were anesthetized using a barbiturate/alcohol preparation $(0.3$ $\mathrm{ml} / 100 \mathrm{gm}$ ). After the rats were marked for identification and shaved, they were placed in the stereotaxic frame (David Kopf, Tujunga, CA), and an incision was made into the scalp to expose the skull. The incisor bar was then adjusted to the level head position. Rats in group BLA ( $n=$ 8) received intracranial injections of a total of $0.5 \mu \mathrm{l}$ of $0.09 \mathrm{M}$ quinolinic acid dissolved in PBS at the following coordinate sites: anteroposterior, -2.3 and -3.0 (two sites); lateral, \pm 4.6 ; ventral, -7.3 . One injection was given on the left and a second on the right side of the midline, using level-head coordinates derived from the stereotaxic atlas of Paxinos and Watson (1998). The injections were made using a $10 \mu \mathrm{l}$ Hamilton syringe through a 30 gauge injection cannula, which was glued into a 23 gauge sleeve for support. The toxin was infused at the rate of $0.05 \mu \mathrm{l} / \mathrm{min}$. The cannula was then left in place for 5 min to allow diffusion of the toxin away from the cannula tip before being raised.

To control for the effects of anesthesia being placed in the stereotaxic instrument and skull holes and the lowering of the injection cannula into the brain, the behavior of the lesioned animals was compared with that of sham operated rats. For the rats in group sham $(n=8)$, exactly the same surgical procedure was conducted but the injection cannula was filled with PBS alone and lowered to the same position as for the GC group, but no fluid was injected.

\section{Histological procedures}

At the end of the experiment, the animals were injected with a lethal barbiturate overdose and perfused transcardially with $0.9 \%$ saline followed by $10 \%$ formalin solution. The brains were stored in $10 \%$ formalin solution for $48 \mathrm{hr}$ before being transferred to a $25 \%$ sucrose solution. Over a period of days the brains were allowed to sink in the sucrose solution, after which $60 \mu \mathrm{m}$ frozen coronal sections were cut throughout the region of the BLA, mounted on glass slides, and stained with cresyl violet. Slides were examined for extent of lesion by microscopically examining sections with reference to the stereotaxic atlas of Paxinos and Watson (1998). Histological assessment was conducted by comparing lesioned brains with the sham brain and by looking for the following features: gross morphological changes such as holes and tissue collapse; the position and extent of gliosis and scarring; the cannula tract and injection placement; and signs of neuronal cell body shriveling and loss.

\section{Behavioral procedures}

Except where indicated, the rats were run twice daily in four squads of four, each squad containing subjects from both lesion conditions, counterbalanced for operant box.

Instrumental acquisition. Three days after the shift to a food deprivation schedule, the behavioral phase began with $3 \mathrm{~d}$ of magazine training in the operant chambers. Pellets and maltodextrin were delivered noncontingently into the magazine on a random time (RT), $30 \mathrm{sec}$ schedule in two separate sessions with both manipulanda withdrawn. Throughout the experiment, each session began with the onset of the house light and terminated with its offset after $20 \mathrm{~min}$. The assessment of instrumental acquisition began on day 4. Action-outcome assignment was counterbalanced such that for four animals in group BLA and four in group Sham, pressing the lever delivered the pellets and pulling the chain delivered the maltodextrin; for the remaining animals in each group, the actionoutcome assignments were reversed. Throughout training the rats were given two separate training sessions each day: one on the lever alone and the other on the chain alone with the action that was trained first on each day alternating from one day to the next. At no stage were the lever pressing and chain pulling actions explicitly shaped by the experimenter. During this phase, animals were trained on a fixed interval (FI) $20 \mathrm{sec}$ schedule, and this training continued on each action until each animal had earned 100 of each outcome, at which point the acquisition phase terminated. The effect of the delivery of each outcome on the number of actions performed before the delivery of the next outcome was used as a measure of the rate of acquisition.

Instrumental training. On the day after the acquisition assessment was 
terminated, animals were trained to lever press and chain pull on a constant probability schedule that delivered the appropriate outcome with a fixed probability for the first response in each second, again with each action trained separately in each session. This probability was 0.25 in the first session, 0.1 in the following two sessions, and 0.05 in the next eight sessions. Action-outcome assignment was the same as that in the acquisition phase. Again, at no stage were the lever pressing and chain pulling actions explicitly shaped by the experimenter. This constant probability schedule approximates to a random ratio (RR) schedule with a mean ratio parameter increasing from 4 , through 10 , to 20 responses.

Outcome devaluation: extinction test. The devaluation treatment was conducted on the day after the final instrumental training session. This was accomplished by prefeeding the rats with one of the two outcomes for $60 \mathrm{~min}$ in the feeding cages. The allocation of the outcome to each rat for the prefeeding phase was counterbalanced within each group both for the action whose outcome was devalued (i.e., lever vs chain), and for the outcome devalued (i.e., pellets vs maltodextrin). Thus, in both group lesion and group sham, for four rats, the lever outcome was devalued, whereas for four rats, the chain outcome was devalued, and for four rats, pellets were devalued, whereas for four rats, maltodextrin was devalued. Immediately after this treatment, the rats were placed in the operant chambers for the 20 min choice extinction test. In this test, both the lever and the chain were available, but neither of the two outcomes was delivered.

Outcome devaluation: reward test. The day after the extinction test, the animals were retrained on the two manipulanda in separate sessions on the RR 20 schedule. On the next day, the rats were given a reward test conducted with both the levers and chains present. This test differed procedurally from the devaluation treatment and extinction test only to the extent that the two outcomes were delivered as a consequence of instrumental performance. In this 20 min test session, the two outcomes were delivered on independent ratio schedules with each outcome earned on an RR 20 schedule (i.e., with a probability of 0.05 ). Before this second test, the rats consumed the same outcome that they had been given before the extinction test for $1 \mathrm{hr}$ in the feeding cages.

\section{Experiment 2}

\section{Subjects and apparatus}

The subjects and apparatus were the same as those used in experiment 1 .

\section{Procedure}

After the reward test of experiment 1, all rats received two sessions of retraining on each of the two manipulanda in separate sessions, with each outcome delivered with a probability of 0.05 for the first response in each second, as in the training phase of experiment 1 . On the following day, the contingency assessment began. The rats continued to be trained on the two manipulanda with the appropriate paired outcome delivered with a probability of 0.05 in separate $30 \mathrm{~min}$ sessions each day. They earned the same outcomes as in experiment 1 , but in addition, one of the two outcomes was also delivered unpaired in each of the sessions, such that one of the action-outcome contingencies was degraded and the other was not. Thus, for each subject the unpaired outcome was the same as the paired outcome in one of the daily sessions and different from the paired outcome in the other session. These unpaired outcomes were also delivered with a probability of 0.05 but after each second without a response. Within each group, the type of unpaired outcome (food pellets versus maltodextrin solution) delivered was counterbalanced with respect to the action-outcome assignment. Thus, for half of the animals trained to lever press for pellets and to chain pull for maltodextrin, the unpaired outcome was pellets, whereas for the other animals it was maltodextrin, and likewise for the animals that earned maltodextrin on the lever and pellets on the chain. The contingency assessment lasted for four sessions with each action and was conducted on successive days. On the fifth day, responding on both the lever and chain was extinguished in separate sessions in the absence of any outcomes.

\section{Experiment 3}

\section{Subjects and apparatus}

Subjects were 20 male Hooded Lister rats housed and maintained under conditions similar to those described in experiment 1 . Of these animals, 10 were given lesions of the BLA exactly as described in experiment 1 , and 10 were given sham surgery. The animals were tested in the operant chambers used in the previous studies with the magazine flap doors fixed in the open position. The animals were maintained on a $22.5 \mathrm{hr}$ food deprivation schedule by being fed for $1.5 \mathrm{hr}$ in their home cages after the daily training session. Tap water was available ad libitum in the home cages.

\section{Procedure: behavioral training}

The animals initially received a session of magazine training in which 30 food pellets were delivered on an RT schedule with the levers and chains retracted. The program that determined this and all other interval contingencies used in this experiment scheduled an available food pellet with a probability of $1 / t$ in each second, where $t$ is the programmed average interpellet interval. There followed two $20 \mathrm{~min}$ instrumental training sessions in which chain pulling and lever pressing were reinforced in separate sessions on a random interval (RI) 2 sec schedule with only the appropriate manipulandum present.

After this pretraining, the rats were introduced to the heterogeneous chain schedule. The first action of this chain was designated as A1 and the second as A2. For five animals in both the BLA group and sham group, chain pulling acted as $\mathrm{A} 1$ and lever pressing acted as $\mathrm{A} 2$, with the remaining animals receiving the opposite assignment. Scheduled food pellets were delivered contingent on the performance of A2, given that A1 had been performed at least once after the food pellet became available. If the rats discriminated between $\mathrm{A} 1$ and $\mathrm{A} 2$, the reinforced chain $\mathrm{A} 1 \rightarrow \mathrm{A} 2$ should have predominated over the other three possible sequences: $\mathrm{A} 1 \rightarrow \mathrm{A} 1, \mathrm{~A} 2 \rightarrow \mathrm{A} 1$, and $\mathrm{A} 2 \rightarrow \mathrm{A} 2$. The parameter of the RI schedule was 2 sec for the first session of chain training and $15 \mathrm{sec}$ for the next three sessions. Both groups received one further session on an RI $30 \mathrm{sec}$ schedule during which performance under the chain contingency was measured. All sessions started when the house lights were turned on and terminated when they were turned off after 30 food pellets had been delivered.

\section{Experiment 4}

\section{Subjects and apparatus}

The subjects and apparatus were those used in experiment 3.

\section{Procedure}

The aim of experiment 4 was to assess the ability of BLA lesioned rats to discriminate between the pellet and maltodextrin outcomes used in the previous experiments. To achieve this, we assessed the performance of the rats used in experiment 3 on a task that required them to use the specific outcome delivered in a particular session to discriminate which of two actions, either lever pressing or chain pulling, was rewarded and which of these actions was not rewarded in that session.

After the assessment of performance on the chain schedule in experiment 3 , all rats were retrained such that they received two training sessions per day during each of which performance on only one of the manipulanda, either the chain or the lever, was reinforced on an RI $30 \mathrm{sec}$ schedule. Performance on the other manipulandum in each session was never rewarded. The action reinforced in the first and second session was alternated across days. Chain pulling was reinforced with the food pellets, and lever pressing was reinforced with the maltodextrin in six of the animals in the BLA group and the sham group, with the remaining animals receiving the opposite assignment. To provide additional exposure to the discriminanda, the type of outcome used to reinforce the rewarded action in any particular session was also delivered on an RT 60 sec schedule in that session. Each of the sessions started when the house lights were turned on and ended after $15 \mathrm{~min}$ when they were turned off. Discrimination training continued for $10 \mathrm{~d}$.

Interval schedules set up reinforcer delivery on the basis of the time 


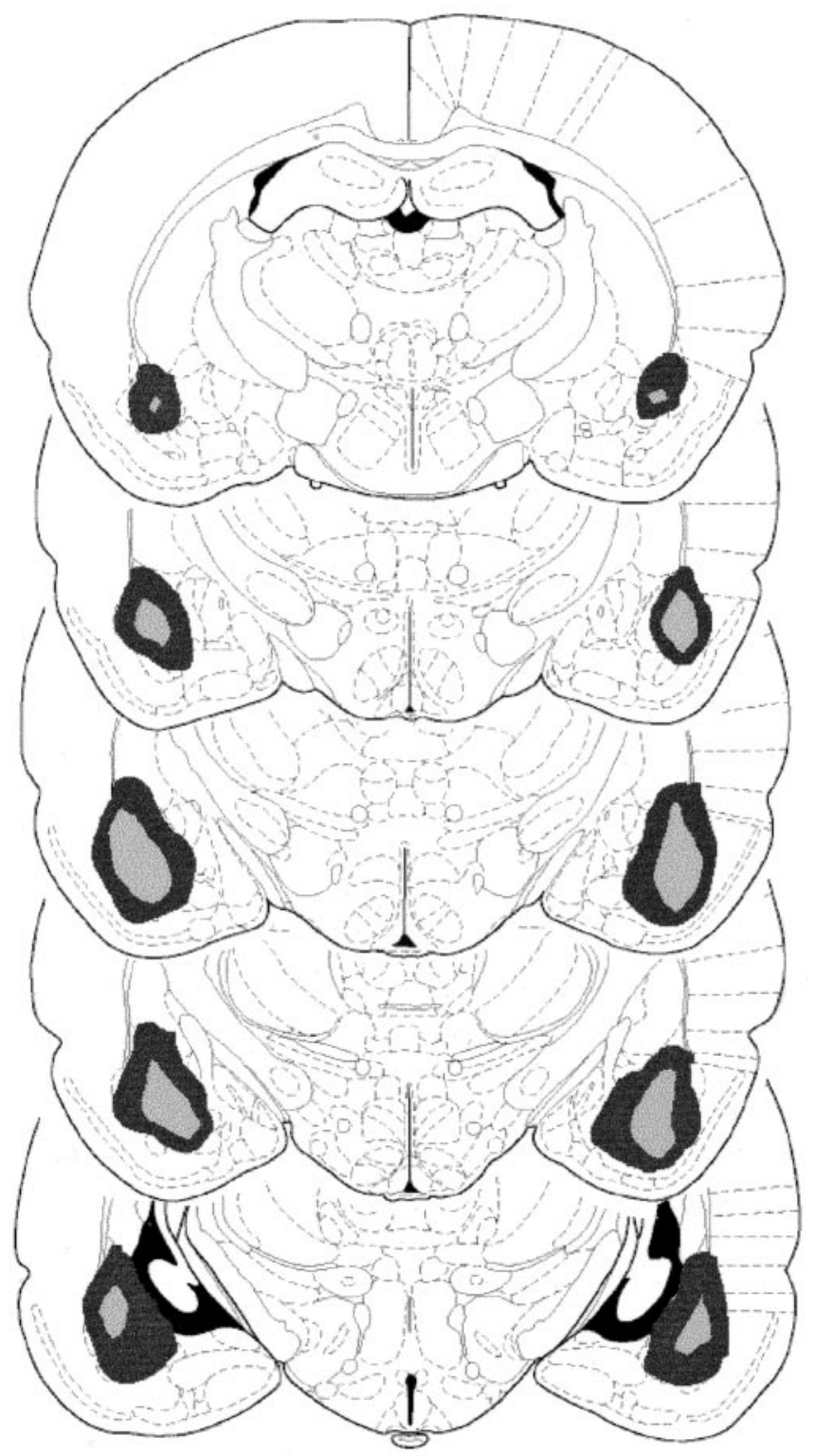

Figure 1. Diagrams of coronal sections $(-1.88,-2.3,-2.8,-3.3,-3.8 \mathrm{~mm}$ posterior to bregma, from top to bottom) on which the extent of cell loss observed after bilateral infusions of quinolynic acid aimed at the BLA has been reconstructed from histology to reveal the largest (darker) and smallest (lighter) regions of damage induced in BLA lesioned animals used in this series of experiments.

since last reward delivery and independently of response rate. Delivery of the reward on a RT schedule is less likely, therefore, to have an effect on the rats' ability to detect the instrumental action-outcome contingency relative to ratio schedules, particularly in a situation in which the rats are asked to discriminate rewarded from nonrewarded actions. As such, this concurrent RT-RI schedule determines that the type of reinforcer delivered in a particular session, whether it was contingent or noncontingent, signals which action was reinforced in that session. As a consequence, successful discrimination performance requires that the rats accurately discriminate the pellet and maltodextrin outcomes.

\section{Results Experiment 1}

Histology

Figure 1 illustrates the largest and smallest areas of lesion damage observed in the BLA lesioned rats used in the current experi-

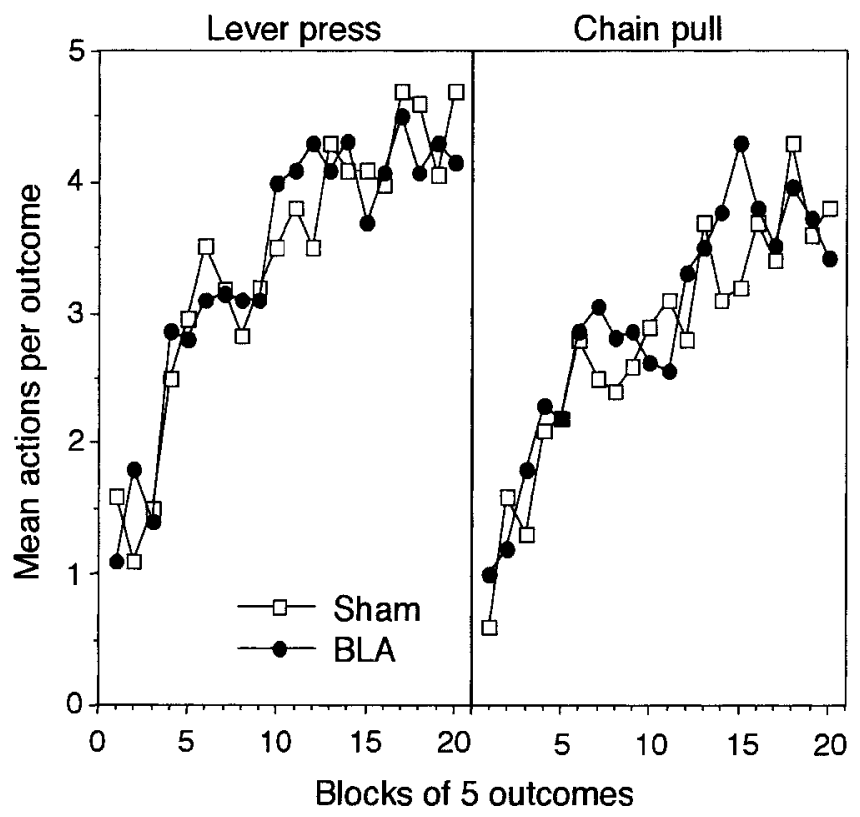

Figure 2. Experiment 1: the mean number of lever presses (left panel) and chain pulls (right panel) performed per outcome during instrumental acquisition on the FI 20 reinforcement schedule used in experiment 1. Data are averaged across blocks of five outcomes and presented separately for group BLA (O) and group Sham ( $\square$ ).

ments. No recovery problem or weight loss was observed after surgery. All but the largest lesions spared the central nucleus, and in no cases was it damaged bilaterally. The entire lateral and basal nuclei were destroyed along their full rostrocaudal extent in all cases. In no instances was there significant damage to the overlying cortex. Some ventricular enlargement at the most caudal extent of the lesions was observed, but again, this was not systematic across animals.

\section{Instrumental acquisition}

The results of the instrumental acquisition phase are presented for the lesioned and sham lesioned rats in Figure 2 separately for the acquisition of lever pressing (left panel) and the acquisition of chain pulling (right panel). In general, it is clear that the FI $20 \mathrm{sec}$ schedule was successful in establishing slow and orderly acquisition of the two instrumental actions as assessed by the number of actions performed in the $20 \mathrm{sec}$ interval after the delivery of each outcome. Both actions were acquired at a similar rate, and the lesion did not have any consistent impact on either the rate of acquisition or the asymptotic level of performance of the two instrumental actions. As such, BLA lesions appear not to affect the general reinforcing impact of the instrumental outcome. Nor was there any evidence of increased generalization between the two actions, something that might be expected if BLA lesions reduced action discriminability. These conclusions were supported by the statistical analysis.

For this analysis, a three-way mixed ANOVA was conducted with a between-subjects factor of group and within-subjects factors of action and of block averaging the performance of each action into blocks of five outcomes. In this and all subsequent analyses, reliability was always assessed against a type I error rate of 0.05 . This analysis did not reveal a main effect of group $(F<1)$ or of action $\left(F_{(1,14)}=1.79\right)$, nor was the group $\times$ action interaction reliable $(F<1)$. A significant effect of block was found $\left(F_{(19,266)}=15.44\right)$, but neither the two-way nor the three-way interaction involving block was reliable (largest $\left.F_{(19,266)}=1.32\right)$. 


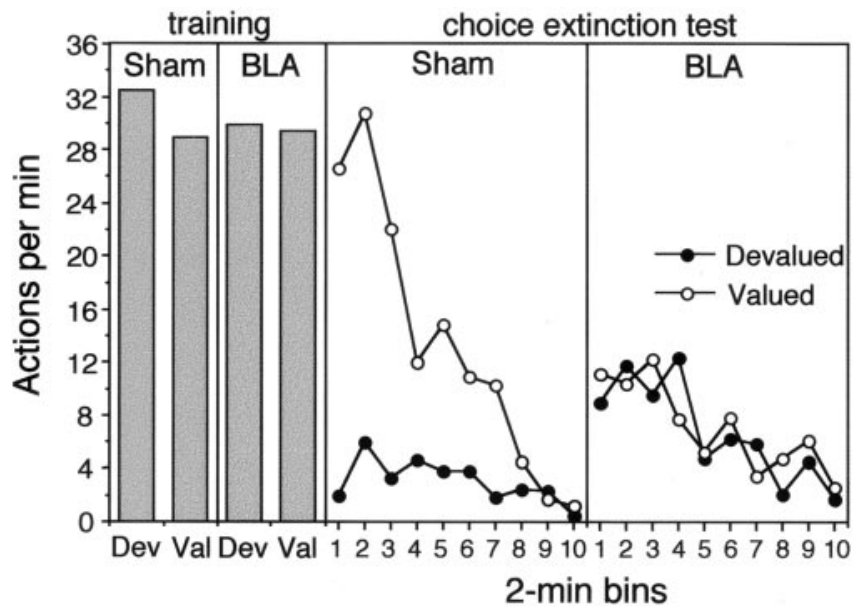

Figure 3. Experiment 1: the number of lever presses and chain pulls (i.e., actions) per minute during instrumental training (left panel) and during the choice extinction test conducted after one of the training outcomes was devalued by a specific satiety treatment. Data from the extinction test are presented for group Sham (center panel) and group BLA (right panel) averaged across 2 min periods with performance of the action that previously delivered the prefed, i.e., Devalued, outcome (O) presented separately from performance of the action that had delivered the non-prefed, i.e., Valued, outcome $(\bigcirc)$ for each group.

Outcome devaluation: extinction test

The results of the extinction test are presented in Figure 3 separately for the action that, in training, delivered the outcome subsequently devalued by specific satiety (i.e., the devalued action) and for the action trained with the outcome that remained valued (i.e., the valued action). The performance on the valued and devalued actions is presented separately for group sham (center panel) and group BLA (right panel). The data from the final training session on the RR 20 schedule are presented in the left panel (see below for discussion). The results from the extinction test for group sham are clear: the performance of the devalued action was markedly reduced compared with that of the valued action. Although performance generally declined over the course of the extinction session, from the very first 2 min period a striking difference in performance was evident. Importantly, group BLA did not show this difference. Indeed, in this group no clear or consistent evidence of a devaluation effect emerged at any point during the extinction test. Again, performance appeared to decline over the course of extinction, but both actions were performed at a similar rate throughout the test.

For the statistical analysis, a three-way mixed ANOVA was conducted with a between-subjects factor of group and withinsubjects factors of devaluation, separating performance on the devalued action from that on the valued action, and of period, separating performance into $2 \mathrm{~min}$ bins. There was no main effect of group $(F<1)$, but both the main effect of devaluation $\left(F_{(1,14)}\right.$ $=22.80)$, and, more importantly, the group $\times$ devaluation interaction $\left(F_{(1,14)}=19.76\right)$ were significant. Simple main effects analysis conducted on the significant interaction revealed that performance on both the valued action and the devalued action differed between groups, with the animals in group BLA performing at a significantly lower rate on the valued action $\left(F_{(1,14)}=4.64\right)$ and a significantly higher rate on the devalued action $\left(F_{(1,14)}=4.92\right)$ than group sham. In addition, although a significant devaluation effect emerged in group sham $\left(F_{(1,14)}=42.51\right)$, no such effect was found in group BLA $(F<1)$. Finally, the overall analysis revealed effects of period $\left(F_{(9,126)}=10.67\right)$, a devaluation $\times$ period interaction $\left(F_{(9,126)}=2.37\right)$, and a significant group $\times$ devaluation $\times$

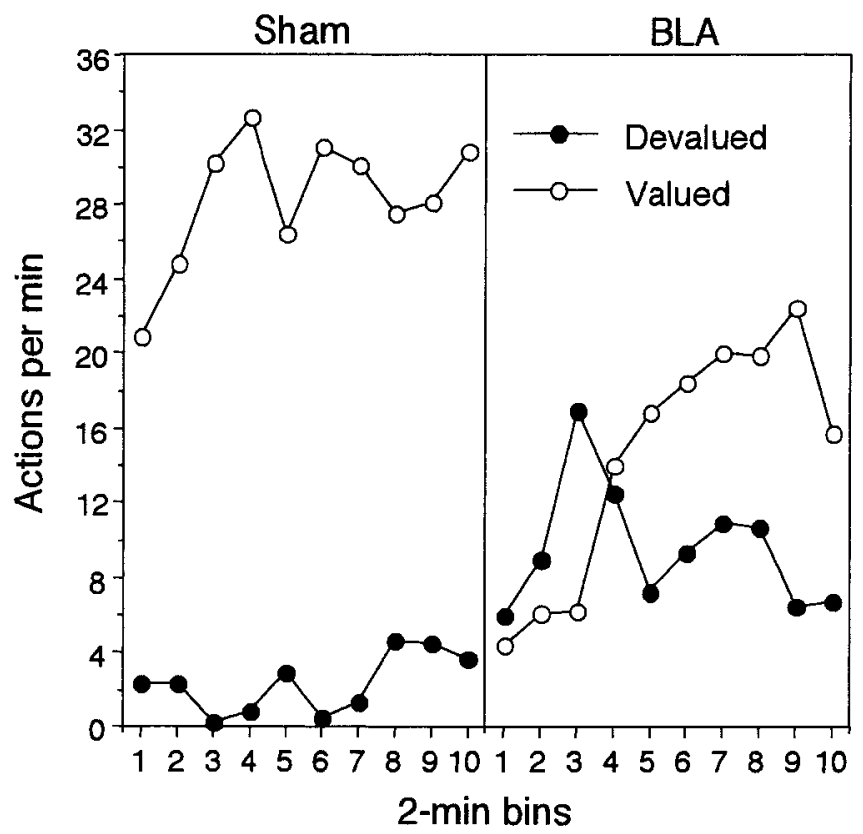

Figure 4. Experiment 1: the number of lever presses and chain pulls (i.e., actions) per minute during the choice reward test conducted after one of the training outcomes was devalued by a specific satiety treatment. In contrast to the extinction test, performance of lever press and chain pull actions delivered the training outcomes on independent random ratio schedules. Data from the reward test are presented for group Sham (left panel) and group BLA (right panel) averaged across 2 min periods with performance of the action that previously delivered the prefed, i.e., Devalued, outcome (O) presented separately from performance of the action that had delivered the non-prefed, i.e., Valued, outcome (O) for each group.

period interaction $\left(F_{(9,126)}=2.37\right)$, confirming that performance generally declined over the extinction session and at a faster rate for the valued than for the devalued action in shams but not in the lesioned rats.

These effects of lesions of the BLA on the outcomedevaluation effect occurred only during the test and were not present in the training data. The data from the final training session on the RR 20 schedule are presented in Figure 3 (left panel). As is clear from that figure, performance between the two groups was very similar as was their performance on the devalued and valued actions. Analysis of these data revealed no main effects of group and devaluation nor any interaction between these factors $(F<1)$.

This effect can also not be attributed to any difference in the amount of pellets and maltodextrin consumed by the two groups during the specific satiety treatment. Sham animals ate $8.3 \mathrm{gm}$ of pellets or drank $15.9 \mathrm{ml}$ of maltodextrin, and BLA lesioned animals ate $8.8 \mathrm{gm}$ of pellets or drank $15.6 \mathrm{ml}$ of maltodextrin during the prefeeding phase. These means did not differ significantly $(F<1)$.

\section{Outcome devaluation: reward test}

The results of the reward test are presented in Figure 4, again separately for devalued and valued actions and for group sham (left panel) and group BLA (rightpanel). As in the extinction test, devaluation of the instrumental outcome induced a strong reduction in the performance of the devalued action in group sham. In contrast, this effect appeared to be much weaker in group BLA and, if anything, emerged only toward the end of the test session as either the action delivering the still valued outcome recovered (but see experiment 2) or the rats learned to avoid the manipulandum paired with the devalued outcome. Certainly, early in the ses- 

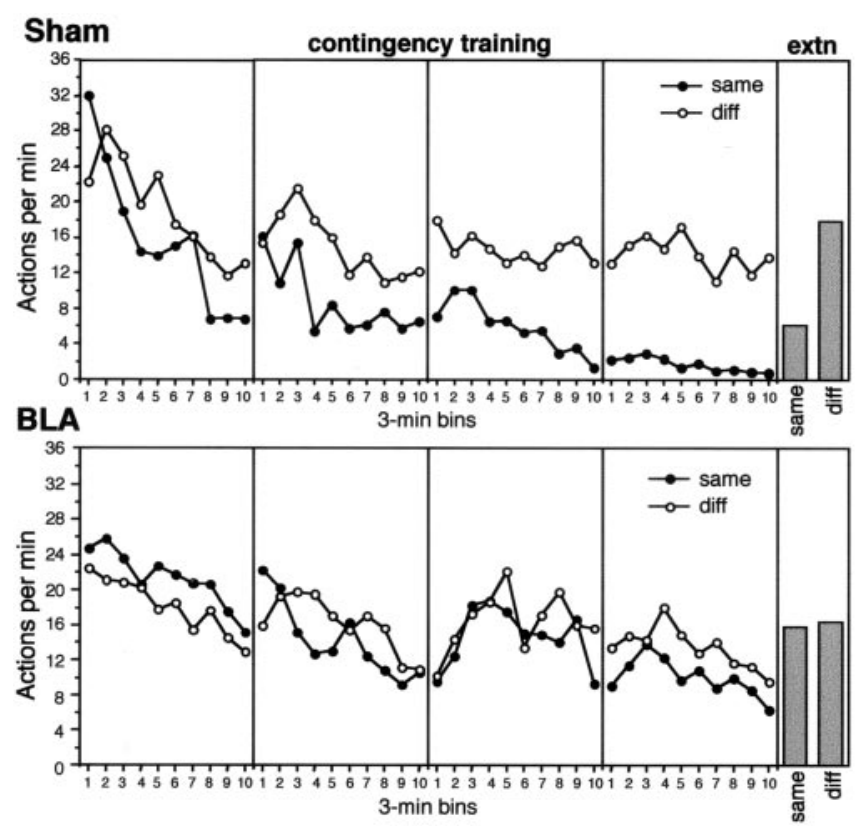

Figure 5. Experiment 2: mean performance of lever press and chain pull actions per minute, averaged over 3 min bins, during each of the $4 \mathrm{~d}$ of contingency assessment (left four panels) and during the extinction test (right panel). Test performance is divided into two panels: the top panels show the data from group Sham and the bottom panels show the data from group BLA. In this figure, performance of each action is presented separately in each panel according to whether the action-outcome contingency has been degraded, i.e., the outcome delivered by performing the action is the same as the one now delivered without performing the action (same, O), or has not been degraded, i.e., the outcome delivered by performing the action differs from that delivered without performing the action $($ diff, $\bigcirc)$. In the panel illustrating the extinction test (extn), the previously degraded action-outcome contingency remains designated as same and the nondegraded as diff, although no outcomes were presented in this test.

sion, there was no evidence of a selective devaluation effect in the BLA lesioned rats. A three-way mixed ANOVA found no effect of group $(F<1)$ but a significant effect of devaluation $\left(F_{(1,14)}=\right.$ 9.89) and a significant group $\times$ devaluation interaction $\left(F_{(1,14)}=\right.$ $4.91)$. Furthermore, there was an effect of period $\left(F_{(9,126)}=2.07\right)$, but no other interactions involving group, devaluation, or period were significant (largest $\left.F_{(9,126)}=1.66\right)$. Simple effects analysis conducted on the significant interaction revealed that there was a reliable devaluation effect in group sham $\left(F_{(1,14)}=14.37\right)$ but not in group BLA $(F<1)$.

Again, this effect of outcome devaluation was found during the test and was not present in the retraining session conducted between the extinction test and the reward test. Comparable analysis of that training session revealed no effect of lesion or of devaluation or any interaction between these factors $(F<1)$. Rate of performance on the devalued and valued actions, respectively, for the two groups was as follows: group sham, 27.6 and 26.1 actions per minute; group BLA, 25.3 and 26.4 actions per minute; this effect was not attributable to any difference in the amount of the pellet and maltodextrin outcomes consumed by the two groups during the specific satiety treatment. On average, sham animals ate $7.9 \mathrm{gm}$ of pellets or drank $15.2 \mathrm{ml}$ of maltodextrin, and BLA lesioned animals ate $8.3 \mathrm{gm}$ of pellets or drank $15.8 \mathrm{ml}$ of maltodextrin during the prefeeding phase. These means did not differ significantly $(F<1)$.

\section{Experiment 2}

The results from the contingency assessment are presented in Figure 5 separately for each of the four sessions of this phase (left

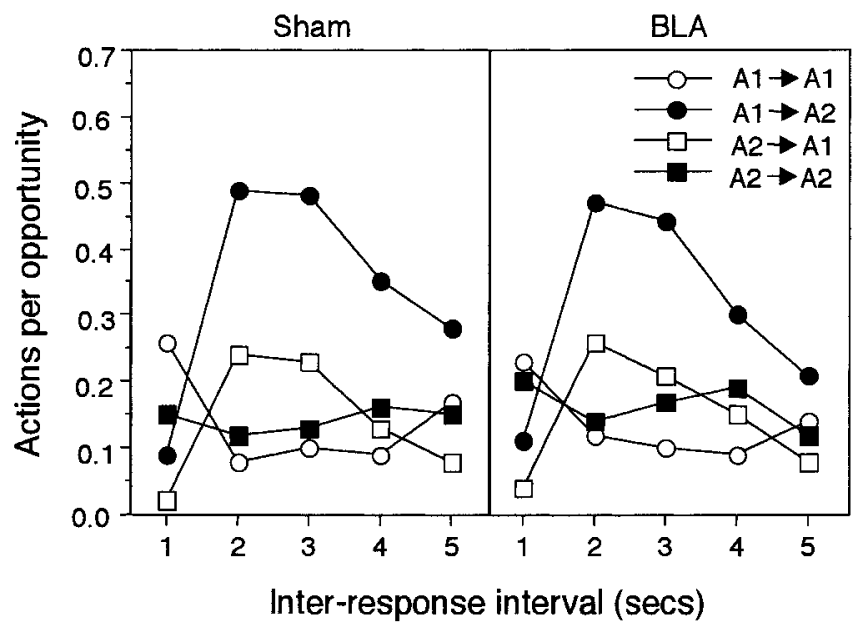

Figure 6. Experiment 3: performance on the heterogeneous chain of instrumental actions presented as actions per opportunity in each second after performance of either the lever press or chain pull actions presented separately for each of the possible orders of these responses and for animals in group Sham (left panel) and group BLA (right panel). For half of the rats in each group, $A 1$ was lever pressing and $A 2$ was chain pulling, whereas for the remaining rats these assignments were reversed.

four panels) and for the extinction test (far right panel). The response rates for group sham (top panels) and group BLA (bottom panels) are presented separately for the actions for which the paired and unpaired outcomes were either the same (same) or different (diff). Over the four sessions of training, the performance of the same action was reduced more than that of the different action in group sham, thereby demonstrating that the action-outcome contingency was successfully degraded by this manipulation. This conclusion was further confirmed in the extinction test in which this pattern of responding clearly persisted when no outcomes were presented. By contrast, the rate of the same and different actions was similar in group BLA, demonstrating that lesioned animals were insensitive to whether the unpaired reinforcers were the same as or different from the paired reinforcers.

This description of the data was confirmed by the statistical analysis. A four-way mixed ANOVA was conducted on the data from the four sessions of contingency assessment with a betweensubjects factor of group and within-subjects factors of contingency, separating performance of the same and different actions, session, and $3 \mathrm{~min}$ periods in each session. This analysis revealed a main effect of contingency $\left(F_{(1,14)}=5.2\right)$, a main effect of group $\left(F_{(1,14)}=6.9\right)$, and a significant group $\times$ contingency interaction $\left(F_{(1,14)}=9.4\right)$. Simple main effects analyses revealed a significant effect of contingency in group sham $\left(F_{(1,14)}=11.6\right)$ but not in group BLA $(F<1)$. In addition, there was a main effect of session $\left(F_{(3,42)}=23.5\right)$ and an interaction between session and contingency $\left(F_{(3,42)}=5.43\right)$, indicating that the effect of contingency developed over sessions, an effect of period $\left(F_{(9,126)}=6.1\right)$, and a session $\times$ period interaction $\left(F_{(9,126)}=3.6\right)$, demonstrating that overall performance declined within a session with this effect being more evident in earlier than in later sessions. None of the other higher order interactions were significant (all $F$ values $<1$ ).

A two-way analysis of the data from the extinction test was conducted using factors of group and contingency. This analysis revealed a main effect of contingency $\left(F_{(1,14)}=7.0\right)$, an effect of group $\left(F_{(1,14)}=4.44\right)$, and a significant group $\times$ contingency interaction $\left(F_{(1,14)}=6.7\right)$. Simple main effects analysis again re- 
vealed a significant effect of contingency in group sham $\left(F_{(1,14)}=\right.$ 12.9) but not in group BLA $(F<1)$.

\section{Experiment 3}

The data of prime interest, which are displayed in Figure 6, concern the probability of completing the $\mathrm{A} 1 \rightarrow \mathrm{A} 2$ sequence, when the performance of $\mathrm{A} 2$ follows shortly after $\mathrm{A} 1$ during the final session, relative to other possible sequences of actions; i.e., $\mathrm{A} 1 \rightarrow \mathrm{A} 1, \mathrm{~A} 2 \rightarrow \mathrm{A} 2$, and $\mathrm{A} 2 \rightarrow \mathrm{A} 1$. This was assessed by recording the number of occasions on which $\mathrm{A} 2$ was the next action performed after an $\mathrm{A} 1$ as a function of the time since the first action and dividing each of these frequencies by the appropriate number of opportunities for performing A2 after A1. Figure 6 shows that for both lesioned groups and their controls the probability of completing the $\mathrm{A} 1 \rightarrow \mathrm{A} 2$ sequence was low immediately after $\mathrm{A} 1$ but then rose to a peak in the second and third second after A1 before declining with longer intervals. Although a somewhat similar profile was seen for the $\mathrm{A} 2 \rightarrow \mathrm{A} 1$ sequence, the overall probability of this sequence was much lower. The profile for repeated action sequences, $\mathrm{A} 1 \rightarrow \mathrm{A} 1$ and $\mathrm{A} 2 \rightarrow \mathrm{A} 2$, showed a small peak in the first second followed by a low, constant probability across the remainder of the recording period. The profiles and overall probability levels for the different sequences were very similar in the lesioned and control animals.

This description is supported by analyses that included two between-subject factors that distinguished between the performance of the sham and BLA lesioned rats and the effect of the assignment of lever pressing and chain pulling on $\mathrm{A} 1$ and $\mathrm{A} 2$, and two within-subject factors that contrasted the performance of the different sequences and the effect of the interval between the actions. This overall analysis revealed a significant effect of sequence type $\left(F_{(3,48)}=35.76\right)$, which interacted with the effect of the interaction interval $\left(F_{(12,192)}=21.00\right)$. Pairwise comparisons by the Newman-Keuls procedure revealed that the overall probability of the $\mathrm{A} 1 \rightarrow \mathrm{A} 2$ sequence was higher than that of all the other action sequences that did not differ significantly. Importantly, there was no evidence that the lesion affected performance of the different possible sequences of actions. There were no significant main effects and no interactions involving the lesion factor (largest $F_{(4,64)}=1.31$ ).

The lesions and the action assignment did not affect the overall rates of performance on the final training session $\left(\right.$ all $F_{(1,16)}<$ 1.62). The number of $A 1$ and $A 2$ actions performed per minute in that session were BLA, 9.2 and 12.1, and sham, 7.9 and 10.6, respectively.

\section{Experiment 4}

Inspection of the relative performance of the two actions during the discrimination established that this was partly determined by an interaction between an animal's response bias toward lever pressing or chain pulling and the discriminative control exerted by the outcome delivered in that session. To determine the discriminative control exerted by the outcome independently of response bias, therefore, performance was categorized in terms of the session on each day that yielded the highest rate for the rewarded action, regardless of whether it was the first or second session of the day. This session was referred to as the maximum $\mathrm{A}+$ session, and performance on this session was compared with that in the other, minimum $\mathrm{A}+$, sessions. The maximum $\mathrm{A}+$ sessions were those in which the dominant response was reinforced, whereas minimum $\mathrm{A}+$ sessions were ones in which the subdominant response was reinforced. Therefore, it is performance in the minimum $\mathrm{A}+$ sessions that most unambiguously
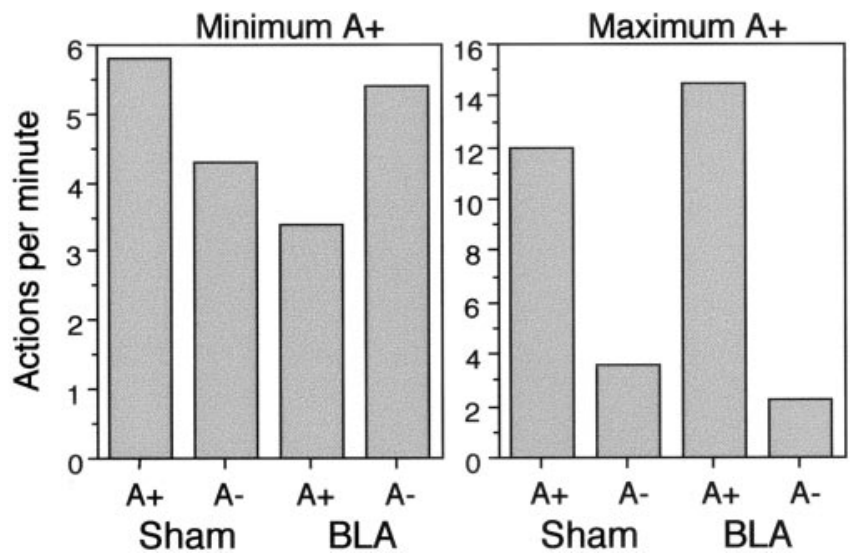

Figure 7. Experiment 4: mean performance of the rewarded $(A+)$ and unrewarded $(A-)$ actions in the outcome discrimination training sessions presented separately for the minimum $\mathrm{A}+($ left panel) and maximum A+ (right panel) sessions and for groups Sham and group BLA.

reveals the degree of discriminative control exerted by the outcome. Figure 7, which displays performance of the sham and BLA groups during these two types of sessions averaged across the $10 \mathrm{~d}$ of training, shows that the sham animals exhibited the appropriate discrimination in both types of session. In both maximum and minimum $\mathrm{A}+$ sessions, they performed the rewarded action $(A+)$ more than the nonreward action $(A-)$, although the magnitude of the discrimination was larger on the maximum A+ sessions. This is the expected pattern if the type of outcome (pellets or maltodextrin) delivered in a session acquires control over the choice between the two actions.

In contrast, the BLA animals exhibited a very different pattern. Although they showed the appropriate discrimination on the maximum $\mathrm{A}+$ sessions, there was no evidence found for the appropriate discrimination on the minimum $\mathrm{A}+$ sessions. In fact the performance pattern was reversed on these latter sessions, with the lesioned rats performing the nonreinforced $\mathrm{A}-$ actions more than the reinforced $\mathrm{A}+$ action. An overall analysis of performance in these sessions revealed a three-way interaction between lesion (BLA vs Sham), the reward contingency (A+ vs $\mathrm{A}-$ ), and the type of session (maximum $\mathrm{A}+$ vs minimum $\mathrm{A}+$ ) $\left(F_{(1,18)}=23.28\right)$. A separate analysis of performance in the maximum $\mathrm{A}+$ session yielded only an interaction with contingency for the lesion factor $\left(F_{(1,18)}=8.23\right)$, reflecting the fact that during these sessions the discrimination was greater for the BLA animals than for the shams. Simple effects analyses revealed, however, that the effect of contingency was reliable for both the $\operatorname{BLA}\left(F_{(1,18)}\right.$ $=161.13)$ and control animals $\left(F_{(1,18)}=74.58\right)$. A corresponding analysis of the minimum $\mathrm{A}+$ session also produced a significant interaction between the lesion and the reinforcement contingency $\left(F_{(1,18)}=21.79\right)$, but one that had a very different source. The sham animals showed a discrimination consistent with the reinforcement contingency in force on the minimum A+ sessions by performing the rewarded action more than the nonrewarded one $\left(F_{(1,18)}=7.96\right)$. By contrast, the BLA animals exhibited a reverse discrimination, performing the nonrewarded action significantly more than the rewarded one $\left(F_{(1,18)}=14.28\right)$. In other words, the performance of the BLA animals on minimum $\mathrm{A}+$ sessions conformed to the reinforcement contingency in effect on the maximum $\mathrm{A}+$ sessions.

The consistent discrimination in the sham animals across both the maximum and minimum $\mathrm{A}+$ sessions strongly suggests that the performance was controlled by the type of outcome de- 
livered in a session. By contrast, the inconsistency shown by the BLA animals in their performance in maximum and minimum $\mathrm{A}+$ sessions is compatible with a failure to discriminate between the two outcomes. In the absence of the reliable cue to the reinforced action, these animals showed an arbitrary preference for one of the actions, the one rewarded on the maximum $\mathrm{A}+$ sessions, that then underwent repeated acquisition and extinction during the successive maximum $\mathrm{A}+$ and minimum $\mathrm{A}+$ sessions, respectively. Hence, the data from experiment 4 provide evidence that BLA lesions attenuate the ability of rats to use the sensory properties of rewarding outcomes to control the performance of their instrumental actions.

\section{Discussion}

As has been reported after lesions of the GC (Balleine and Dickinson, 2000), BLA lesions were found to produce a clear deficit in the sensitivity of instrumental performance to post-training changes in the incentive value of the instrumental outcome. The effect of GC lesions was limited, however, to choice performance assessed in an extinction test, whereas the effect of BLA lesions was observed both in extinction and in a test in which both the valued and devalued rewards were delivered contingent on instrumental performance. As such, although the effects of GC lesions appeared to be limited to the ability of rats to freely recall changes in incentive value, the current data suggest that BLA lesions affect the ability of animals to encode those changes, most likely because of a deficit in encoding the motivational significance associated with sensory features of instrumental outcomes. Thus, although the rats were able to discriminate the performance of lever pressing from chain pulling, both during the acquisition of these actions in experiment 1 and when they were required to perform these actions in series to gain access to reward (experiment 3 ), they appeared to be unable to learn to discriminate which of these actions was rewarded and which was unrewarded when the pellet and maltodextrin outcomes were used as discriminative stimuli (experiment 4).

This deficit in outcome encoding was manifest, in the earlier tests, as a dysfunction in choice performance after outcome devaluation (experiment 1 ) and insensitivity to degradation of the instrumental contingency (experiment 2). Generally, these results established that the lesioned rats were unable to modify their instrumental performance in any selective manner when they were asked either to recall or, indeed, merely to recognize a change in the relationship between the performance of an action and the delivery of a specific rewarding event. Hence, when one outcome was devalued by a specific satiety procedure, BLA lesioned rats failed to modify their performance in either the choice extinction test or the choice reward test. Furthermore, lesioned rats appeared to be insensitive to changes in the causal consequences of their actions; in a situation in which the delivery of one of the two outcomes was equally probable whether its associated action was performed or not, BLA lesioned rats continued to perform both actions at similar and high rates.

It is worth noting that despite these striking and quite specific deficits on tests of action-outcome encoding, the lesioned rats remained sensitive, at least to some degree, to the reinforcing impact of outcome delivery. Thus, in experiment 1 and, indeed, throughout this series, no evidence of a deficit was found either in the acquisition of instrumental conditioning or in the subsequent rate of performance of the instrumental actions. The conclusion that appears to be demanded by these data, therefore, is that although lesioned rats were unable to represent specific outcomes (and so were unable to encode specific action-outcome associa- tions), nevertheless these outcomes were able to engage a general reinforcement mechanism. This may seem a surprising conclusion, but it is not without precedent. When instrumental actions are overtrained or are trained on interval schedules of reinforcement, performance has been reported to change from being goal directed to being more automatic or habitual; i.e., their performance appears similar to that of the BLA lesioned rats in the current experiments and is no longer sensitive to either outcome devaluation (Dickinson et al., 1994) or manipulations of the instrumental contingency (Dickinson et al., 1998). An analysis of the effects of overtraining has been developed on the basis of the argument that two different learning processes contribute to instrumental performance: one involving action-outcome encoding and a second reflecting control by a stimulus-response (S-R) reinforcement process. This latter process is sensitive only to the reinforcing impact of instrumental outcomes and is thought to be driven by a contiguity-based rather than an error-correcting learning rule [cf. Dickinson (1994) for discussion]. Given the behavioral similarities between the effects of BLA lesions and overtraining, it is possible that in the absence of the capacity to encode the action-outcome association, the BLA rats acquired instrumental performance through this second, S-R reinforcement process. Recent evidence (Blundell et al., 2001) suggests, however, that if this is the case, the target response of the acquired $\mathrm{S}-\mathrm{R}$ relation may not be a highly specified behavioral response but a generalized emotional response. At this level, there is little difference between postulating the acquisition of a generalized emotional S-R association and the acquisition of an action- or stimulus-outcome association in which the outcome is represented in terms of the general motivational properties of the reward and not its specific sensory aspects (Konorski and Jerzy, 1967).

A similar claim has been advanced on the basis of the pattern of deficits in Pavlovian conditioning observed after BLA lesions. Numerous reports have noted that damage to the amygdala produces wide-ranging changes in emotional responses to both rewarding events and signals that predict rewarding events in both humans (Adolphs et al., 1998; Morris et al., 1998) and nonhuman primates (Gaffan and Harrison, 1987; Gallagher and Chiba, 1996; Malkova et al., 1997; Baxter et al., 2000). Here two strong traditions have emerged. In aversive learning procedures, lesions of various subnuclei of the amygdala, including the BLA, have been shown to disrupt fear conditioning in various procedures such as conditioned freezing (Maren et al., 1996; LeDoux, 1996, 2000), fear-potentiated startle (Sananes and Davis, 1992), and an operant conditioned punishment task (Killcross et al., 1997), leading to the suggestion that the BLA is involved in the formation of conditioned stimulus (CS)-reinforcer associations that achieve diverse behavioral expression via multiple outputs.

In the appetitive domain, a similar conclusion has been advanced on the basis of evidence that lesions of the BLA attenuate conditioned place preferences for food or drugs of abuse as well as the acquisition of responses associated with a conditioned reinforcer (Everitt and Robbins, 1992; White and McDonald, 1993). In line with these findings, infusion of the NMDA antagonist AP-5 into the BLA has been reported to produce a deficit in lever press acquisition when the performance of that response produces an explicit conditioned reinforcer (i.e., house light off plus a red signal light) presented in addition to, and for $3 \mathrm{sec}$ before, the delivery of a sucrose pellet reward (Baldwin et al., 2000). In addition to these effects, lesions of the BLA have been reported to produce deficits in both second-order conditioning and Pavlovian reinforcer devaluation (Hatfield et al., 1996) and have re- 
cently been reported to produce highly specific deficits in both the differential outcomes effect in discrimination learning and Pavlovian-instrumental transfer (Blundell et al., 2001). These findings suggest that the BLA is involved in the associative learning processes that allow access of the CS to the specific incentive or hedonic properties of their associated rewards and, in common with the current data, have been interpreted as implying that Pavlovian conditioned responding in BLA-lesioned animals is mediated by an associative structure other than a well specified CS-unconditioned stimulus (US) association and involves a more general emotional S-R process [cf. Blundell et al. (2001) for discussion].

An important feature of our argument is the suggestion that it is as a consequence of the role of BLAs in establishing the rewardrelated properties of the outcome in instrumental conditioning that BLA lesions act to affect encoding of the action-outcome association. Specifically, the deficit in rats with BLA lesions appears to be best characterized as a deficit in encoding the sensoryspecific aspects of motivationally significant stimuli. There is now considerable evidence suggesting that these sensory aspects of rewarding events play an important role in the hedonic evaluation of nutritive instrumental outcomes on the basis of their palatability (Balleine, 2001; Berridge, 2001). Hence, to use a recently useful heuristic, it would seem that the basolateral amygdala is involved in aspects of "liking" rather than "wanting" (Simbayi et al., 1986; Berridge, 2001). From this perspective, it appears likely that within the larger system controlling instrumental conditioning, the BLA is a critical component of the process through which outcome value is integrated within the action-outcome association to guide performance.

It is of interest, therefore, that in addition to the BLA, there is a significant body of evidence suggesting that a region of ventrolateral prefrontal cortex comprising dorsal agranular insular and lateral orbital cortices is also involved in the integration of sensory qualities of foods and fluids with the affective and motivational properties of those commodities. For example, lesions of this area cause acute aphagia and reductions in body weight (Kolb, 1974; Kolb et al., 1977), produce deficits in complex olfactory discriminations (Otto and Eichenbaum, 1992), and impair US devaluation effects in a Pavlovian conditioning preparation (Gallagher et al., 1999). The connectivity of the lateral orbital cortices also suggests a role in sensory and affective integration. Reciprocal connections between the gustatory and more rostral agranular insular cortex and the BLA have been described (Sripanidkulchai et al., 1984; Yamamoto et al., 1984), raising the possibility that these areas form a distributed outcome memory relating the affective significance of instrumental outcomes to their sensory features. Interestingly, representations of sensory events in the primate orbitofrontal, but not insular, cortex have been reported to undergo remodeling on the basis of their motivational significance; the responsiveness of neurons in this region sensitive to food or water delivery, as well as to signals for the delivery of these commodities, has been found to be a direct function of the animals' motivational state (e.g., of their degree of food or water deprivation) (cf. Rolls, 1989, 2000). These regions of insular cortex and the BLA have strong connections with the core of the nucleus accumbens, a region that has long been implicated in reward. Lesions of this region have been found to induce a deficit in instrumental outcome devaluation similar to that induced by BLA lesions (Corbit et al., 2001), and hence it is possible that, together, these structures make up the essential circuit through which the incentive properties of the instrumen- tal outcome are integrated with the action-outcome association, a hypothesis that suggests a fruitful avenue for future research.

\section{References}

Adolphs R, Tranel D, Damasio AR (1998) The human amygdala in social judgement. Nature 393:470-474.

Baldwin AE, Holahan MR, Sadeghian K, Kelley AE (2000) N-methyl-Daspartate receptor-dependent plasticity within a distributed corticostriatal network mediates appetitive instrumental learning. Behav Neurosci $114,84-98$.

Balleine BW (1992) The role of incentive learning in instrumental performance following shifts in primary motivation. J Exp Psychol Anim Behav Proc 18:236-250.

Balleine BW (2001) Incentive processes in instrumental conditioning. In: Handbook of contemporary learning theories (Mowrer R, Klein S, eds), pp 307-366. Hillsdale, NJ: LEA.

Balleine BW, Dickinson A (1994) Role of cholecystokinin in the motivational control of instrumental action in rats. Behav Neurosci 108:590-605.

Balleine BW, Dickinson A (1998a) The role of incentive learning in instrumental outcome revaluation by sensory-specific satiety. Anim Learn Behav 26:46-59.

Balleine BW, Dickinson A (1998b) Goal-directed instrumental action: contingency and incentive learning and their cortical substrates. Neuropharmacology 37:407-419.

Balleine BW, Dickinson A (2000) Effect of lesions of the insular cortex on instrumental conditioning: evidence for a role in incentive memory. J Neurosci 20:8954-8964.

Balleine BW, Garner C, Dickinson A, Gonzalez F (1995) Motivational control of heterogeneous instrumental chains. J Exp Psychol Anim Behav Proc 21:203-217.

Baxter MG, Parker A, Lindner CC, Izquierdo AD, Murray EA (2000) Control of response selection by reinforcer value requires interaction of amygdala and orbito-prefrontal cortex. J Neurosci 20:4311-4319.

Berridge KC (2001) Reward learning: reinforcement, incentives, and expectations. In: The psychology of learning and motivation: advances in research and theory, Vol 40 (Medin DL, ed), pp 223-278. San Diego: Academic.

Blundell P, Hall G, Killcross AS (2001) Lesions of the basolateral amygdala disrupt selective aspects of reinforcer representation in rats. J Neurosci 21:9018-9026.

Corbit L, Muir J, Balleine BW (2001) The role of the nucleus accumbens in instrumental conditioning: evidence for a functional dissociation between accumbens core and shell. J Neurosci 21:3251-3260.

Corbit LH, Balleine BW (2000) The role of the hippocampus in instrumental conditioning. J Neurosci 20:4233-4239.

Dickinson A (1994) Instrumental conditioning. In: Animal learning and cognition (Mackintosh NJ, ed), pp 45-79. San Diego: Academic.

Dickinson A, Balleine B (1994) Motivational control of goal-directed action. Anim Learn Behav 22:1-18.

Dickinson A, Balleine BW, Watt A, Gonzales F, Boakes RA (1994) Overtraining and the motivational control of instrumental action. Anim Learn Behav 22:197-206.

Dickinson A, Squire S, Varga Z, Smith JW (1998) Omission learning after instrumental pretraining. Q J Exp Psychol 51B:271-286.

Everitt BJ, Robbins TW (1992) Amygdala-ventral striatal interactions and reward-related processes. In: The amygdala: neurobiological aspects of emotion, memory, and mental dysfunction (Aggleton JP, ed), pp 401429). New York: Wiley.

Gaffan D, Harrison S (1987) Amygdalectomy and disconnection in visual learning for auditory secondary reinforcement by monkeys. J Neurosci 7:2285-2292.

Gallagher M, Chiba AA (1996) The amygdala and emotion. Curr Opin Neurobiol 6:221-227.

Gallagher M, McMahan RW, Schoenbaum G (1999) Orbitofrontal cortex and representation of incentive value in associative learning. J Neurosci 19:6610-6614.

Gallo M, Roldan G, Bures J (1992) Differential involvement of gustatory insular cortex and amygdala in the acquisition and retrieval of conditioned taste aversion in rats. Behav Brain Res 52:91-97. 
Hatfield T, Han JS, Conley M, Gallagher M, Holland PC (1996) Neurotoxic lesions of basolateral, but not central, amygdala interfere with Pavlovian second-order conditioning and reinforcer devaluation effects. J Neurosci 16:5256-5265.

Hull CL (1943) Principles of behavior. New York: Appleton.

Killcross AS, Everitt BJ, Robbins TW (1997) Different types of fear-related behaviour mediated by separate nuclei within amygdala. Nature 388:377-380

Kolb B (1974) Prefrontal lesions alter eating and hoarding behavior in rats. Physiol Behav 12:507-511.

Kolb B, Whishaw IQ, Schallert T (1977) Aphagia, behavior sequencing and body weight set point following orbital frontal lesions in rats. Physiol Behav 19:93-103.

Konorski Jerzy (1967) Integrative activity of the brain: an interdisciplinary approach. Chicago: University of Chicago.

LeDoux J (1996) The emotional brain: the mysterious underpinnings of emotional life. New York: Touchstone.

LeDoux J (2000) The amygdala and emotion: a view through fear. In: The amygdala: a functional analysis (Aggleton JP, ed), pp 289-310. New York: Oxford UP.

Malkova L, Gaffasn D, Murray E (1997) Excitotoxic lesions of the amygdala fail to produce impairment in visual learning for auditory secondary reinforcement but interfere with reinforcer devaluation effects in rhesus monkeys. J Neurosci 17:6011-6020.

Maren S, Aharonov G, Stote DL, Fanselow MS (1996) N-methyl-Daspartate receptors in the basolateral amygdala are required for both acquisition and expression of conditional fear in rats. Behav Neurosci 110:1365-1374.
Morris JS, Ohman A, Dolan RJ (1998) Conscious and unconscious emotional learning in the human amygdala. Nature 393:467-470.

Otto T, Eichenbaum H (1992) Complementary roles of the orbital prefrontal cortex and the perirhinal-entorhinal cortices in an odor-guided delayed-non-matching-to-sample task. Behav Neurosci 106:762-775.

Paxinos G, Watson C (1998) The rat brain in stereotaxic coordinates, Ed 4. Sydney: Academic.

Rescorla RA (1990) Instrumental responses become associated with reinforcers that differ in one feature. Anim Learn Behav 18:206-211.

Rolls ET (1989) Information processing in the taste system of primates. J Exp Biol 146:141-164.

Rolls ET (2000) The orbitofrontal cortex and reward. Cereb Cortex 10:284-294.

Sananes CB, Davis M (1992) N-methyl-D-aspartate lesions of the lateral and basolateral nuclei of the amygdala block fear-potentiated startle and shock sensitization of startle. Behav Neurosci 106:72-80.

Simbayi LC, Boakes RA, Burton MJ (1986) Effects of basolateral amygdala lesions on taste aversions produced by lactose and lithium chloride in the rat. Behav Neurosci 100:455-465.

Sripanidkulchai K, Sripanidkulchai B, Wyss JM (1984) The cortical projection of the basolateral amygdaloid nucleus in the rat: a retrograde fluorescent dye study. J Comp Neurol 229:419-431.

White NM, McDonald RJ (1993) Acquisition of a spatial conditioned place preference is impaired by amygdala lesions and improved by fornix lesions. Behav Brain Res 55:269-281.

Yamamoto T, Azuma S, Kawamura Y (1984) Functional relations between the cortical gustatory area and the amygdala: electrophysiological and behavioral studies in rats. Exp Brain Res 56:23-31. 